\title{
MULTI-SCALE ANALYSIS OF SKIN HYPER-PIGMENTATION EVOLUTION
}

\author{
Sylvain Prigent ${ }^{1}$, Xavier Descombes $^{1}$, Didier Zugaj $^{2}$, Laurent Petit $^{2}$, Josiane Zerubia ${ }^{1}$ \\ ${ }^{1}$ INRIA/I3S, 2004 route des Lucioles, BP93, 06902 Sophia Antipolis, Cedex, France \\ email: Sylvain.Prigent@inria.fr, Xavier.Descombes@inria.fr, Josiane.Zerubia@inria.fr \\ ${ }^{2}$ Galderma R\&D, 2400 Route des Colles, BP87, 06902 Sophia-Antipolis, Cedex, France \\ email: Didier.Zugaj@galderma.com,Laurent.Petit@galderma.com
}

\begin{abstract}
In this paper, we use statistical inference and muti-spectral images to quantify the evolution of skin hyper-pigmentation lesions under treatment. We show that statistical inference allows getting change maps of the disease which can be useful for dermatologists to analyze the disease evolution. Indeed, a local change map is obtained by computing the deviation between two multi-spectral images in a region of interest (ROI). Then, we normalize the obtained map and develop a statistical inference framework to quantify the changes. Finally, we propose a criterion that integrates change maps in order to quantify the treatment efficacy on a patient.
\end{abstract}

Index Terms - multi-scale analysis, statistical inference, multi-spectral image, skin, hyper-pigmentation.

\section{INTRODUCTION}

In dermatology, spectral information is used to quantify the severity of pigmentation lesions like melasma. To this end, spectro-colorimeters allow the measurement of an average spectrum on a small skin area. Such measurements can be repeated to get an evolution curve per patient, or to do statistical analysis of a treatment efficacy on a group of patients. The lack of spatial information about the lesion is the weak point of spectro-colorimeters. To overcome this limit we propose to use multi-spectral images. Multi-spectral images are series of gray scale frames taken at different wavelengths. For hyper-pigmentation, the range of the wavelengths is from $400 \mathrm{~nm}$ to $900 \mathrm{~nm}$.

To characterize hyper-pigmentation lesions, we decompose the image analysis into three steps: the first step is the lesion segmentation. This segmentation can be done manually, with methods based on physical model [1], on CIE $L^{*} a^{*} b$ decomposition [1], or on independent component analysis (ICA) [2]. We proposed a method based on support vector machine (SVM) in [2]. The second step is to design a spectral signature of the lesion. Such a signature can be empirical, for example the Luminance of the CIE $L^{*} a^{*} b$ decomposition, or a spectrum obtained by physical analysis [1]. We proposed in [3] a statistical approach based on ICA and on a function optimisation to obtain a spectral signature. The third step is evaluating the lesion spatial changes between two measurements, which is the purpose of this paper. To perform this evaluation, we have a series of multi-spectral images $\left[H S_{0}, \ldots H S_{k}, \ldots H S_{n}\right]$ taken at times $\left[t_{0}, \ldots t_{k}, \ldots t_{n}\right]$. Moreover, we have a binary classification mask $M_{p_{0}}$ defining the ROI computed on $H S_{0}$ during the first step, and a spectral signature that allows the integration of the multi-spectral images into gray scale images $\left[I_{0}, \ldots I_{k}, \ldots I_{n}\right]$. Thereby, we aim to use the Statistical Parametric Mapping (SPM) methodology [4] based on statistical inference to map the changes between $I_{0}$ and each of the $I_{k}$ images inside the ROI.

The paper is organized as follows. Section 2 describes the statistical inference to quantify local significance of changes. Section 3 proposes a homogeneity criterion to integrate a change map into a scalar criterion. Finally, we demonstrate experimental results on patients whose lesions of melasma have a decreasing severity.

\section{STATISTICAL INFERENCE}

For skin pigmentation analysis, dermatologists can be interested by both height and low intensity changes inside a lesion. In fact, we are interested to evaluate if a treatment affect the pathological area by decreasing the pigmentation in localized spots, or affect large areas. To this end we propose to use statistical inference. Such a methodology was used for fMRI studies on brain activity [4] to detect small intensity but spatially consistent changes and height intensity changes with a multi-scale analysis called SPM. We adapt this framework to our specific detection problem considering a change map defined by $C_{k}=I_{k}-I_{0}$.

\subsection{Change map normalisation}

Statistical inference is a mathematical model for highly correlated Gaussian random field. Two transformations are then need for $C_{k}$ to fit the model. First, we smooth $C_{k}$ with a Gaussian convolution filter to increase it spatial correlation. Second, the ROI of $C_{k}$ denoted $C_{k}^{M_{p_{0}}}$ is defined by a unique 
class on a radiometric image. Thus, $C_{k}^{M_{p_{0}}}$ histogram can be model as monomodal. We use a histogram specification to transform $C_{k}^{M_{p_{0}}}$ histogram into a Gaussian distribution. We center and reduce the obtained distribution to standardize it. In the following we will denote $C_{k}^{N}$ the normalized change map.

\subsection{Change map thresholding}

$C_{k}^{N}$ satisfies the conditions to apply the Gaussian field theory [5]. An important characteristic of a Gaussian field $F$ of size $S$ is the DT (Differential Topology) characteristic defined by:

$$
\chi\left(A_{u}\right)=(-1)^{(D-1)} \sum_{k=0}^{D-1}(-1)^{k} \chi_{k}\left(A_{u}\right),
$$

where the excursion set $A_{u}$ is defined by:

$$
A_{u}=\{s \in S, F(s) \geq u\},
$$

where $u$ is a given threshold, $D$ the dimension of the field and $\chi_{k}\left(A_{u}\right)$ the number of points $s \in A_{u}$ satisfying the conditions: (a) $F(s)=u$, (b) $F^{(i)}(s)=0, \forall i \in[1, D-1]$, (c) $F^{(D)}(s)>0$ and (d) the $(D-1) \times(D-1)$ matrix of second order partial derivatives of $\mathrm{F}(\mathrm{s})$ has exactly $\mathrm{k}$ negative eigenvalues. In the above expressions, $F^{(i)}$ denotes the $i^{\text {th }}$ derivative of $F$. In the 2D case (i.e. $D=2$ ), for a given threshold $u$, the expectation of the DT characteristic of a centred and reduced Gaussian field can be written as [5]:

$$
\mathbb{E}_{\chi\left(A_{u}\right)}=\mathcal{S}(2 \pi)^{\frac{3}{2}}|\Lambda|^{\frac{1}{2}} \sigma^{-3} u e^{-\frac{u^{2}}{2 \sigma^{2}}}
$$

with $\mathcal{S}$ the area of the field, $\Lambda$ the $2 \times 2$ correlation matrix of the field derivatives, and $\sigma^{2}=\mathbb{E}\left(F^{2}\right)$.

In order to estimate the likelihood of a pixel of $C_{k}^{N}$ and its neighborhood under the Gaussian field assumption, we compare its characteristic at a threshold $u$ to the DT characteristic. Two parameters can be used to compute this statistic: the maximum intensity and the spatial extent of the considered neighborhood above $u$.

Let $x_{0}$ denote the maximum intensity of $R_{u}^{x_{0}}$, a region of $C_{k}^{N}$ above the threshold $u$. The likelihood of this region under the Gaussian field assumption is [6]:

$$
P\left(R_{u}^{x_{0}} \in F\right)=\frac{\mathbb{E}_{\chi\left(A_{x_{0}}\right)}}{\mathbb{E}_{\chi\left(A_{u}\right)}}=\frac{x_{0}}{u} e^{\frac{u^{2}-x_{0}^{2}}{2}} .
$$

Let $S_{0}$ denote the spatial extent of $R_{u}^{S_{0}}$, a region of $C_{k}^{N}$ above the threshold $u$. The expectation for this region under the Gaussian field assumption is [6]

$$
\mathbb{E}\left(R_{u}^{S_{0}} \in F\right)=\frac{\mathbb{E}\left(N_{u}\right)}{\mathbb{E}_{\chi\left(A_{u}\right)}},
$$

where $\mathbb{E}\left(N_{u}\right)$ is the expectation to have $N_{u}$ pixels above the threshold $u$ in the random field $F$. As $F$ follows a standard

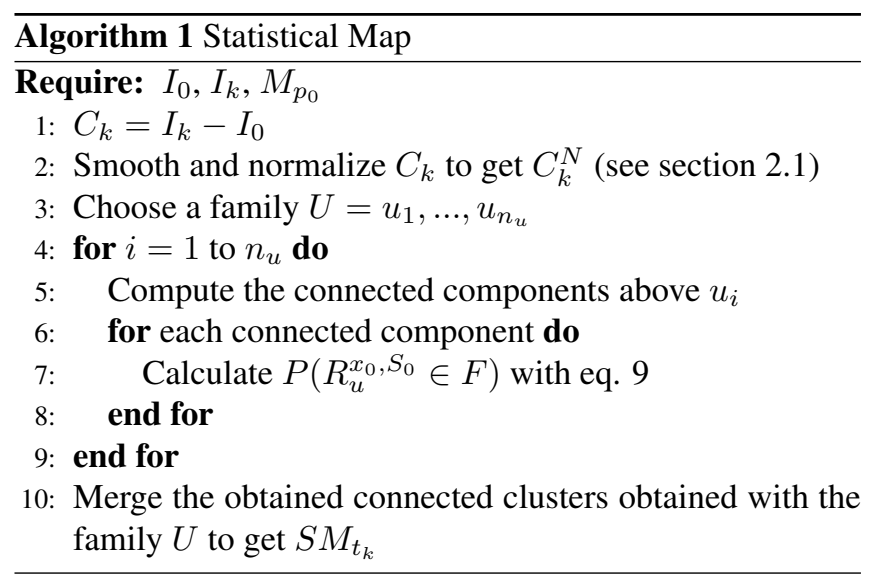

normal distribution,

$$
\mathbb{E}\left(N_{u}\right)=\mathcal{S} \int_{u}^{\infty}(2 \pi)^{\frac{1}{2}} e^{-\frac{x^{2}}{2}}=\mathcal{S} \Phi(-u)
$$

then,

$$
\mathbb{E}\left(R_{u}^{S_{0}} \in F\right)=\frac{\Phi(-u)}{(2 \pi)^{\frac{3}{2}}|\Lambda|^{\frac{1}{2}} u e^{-\frac{u^{2}}{2}}} .
$$

Thus, the number of pixels in a region above the threshold $u$ has an exponential distribution with the parameter $\lambda_{u}=$ $1 / \mathbb{E}\left(R_{u}^{S_{0}} \in F\right)$. We can then write the probability of a region $R_{u}^{S_{0}}$ to appear in a realization of the Gaussian field by:

$$
P\left(R_{u}^{S_{0}} \in F\right)=e^{-\lambda_{u} S_{0}}=e^{\frac{(2 \pi)^{\frac{3}{2}} \mid \Lambda \Lambda^{\frac{1}{2}} S_{0} u e^{-\frac{u^{2}}{2}}}{\Phi(-u)}} .
$$

Finally, for a region of $C_{k}^{N}$ above the threshold $u$ with the maximum intensity $x_{0}$ and the spatial extent $S_{0}$, the probability of $C_{k}^{N}$ to be a realisation of $F$ is [6]:

$$
P\left(R_{u}^{x_{0}, S_{0}} \in F\right)=\min \left(P\left(R_{u}^{x_{0}} \in F\right), P\left(R_{u}^{S_{0}} \in F\right)\right) .
$$

Thereby, to detect changes between $I_{0}$ and $I_{k}$ we define a family of $n_{u}$ thresholds $U=\left\{u_{1}, \ldots, u_{n_{u}}\right\}$. For each $u_{i}, C_{k}^{N}$ is segmented into regions denoted $R_{u_{i}}^{x o, S_{0}}$. This segmentation is obtained by a connected components analysis of the class defined by the pixels of $C_{k}^{N}$ above the threshold $u_{i}$. Then, we assign a probability $P\left(R_{u_{i}}^{x o, S_{0}} \in F\right)$ to each region $R_{u_{i}}^{x o, S_{0}}$. The superposition of the obtained regions for each threshold gives $S M_{t_{k}}$, a statistical map of the changes (Figs. 2 and 3).

The algorithm 1 summarizes the procedure for obtaining $S M_{t_{k}}$ between $I_{0}$ and $I_{k}$ for a given patient.

\subsection{Rare event hypothesis}

The statistical approach proposed above makes the rare events hypothesis. In studied images, a large region of the ROI can change if the disease decreases significantly. Thus, if we apply the methodology described above, only a small amount of the changes will be quantified and the resulting homogeneity 
criterion will be difficult to analyze. We then introduce the normalisation:

$$
F_{t_{k} / 0}^{N_{d} e}=F_{t_{k / 0}}^{N e}+\frac{1}{N_{r}} \sum_{r=t_{0}}^{t_{k}}\left(\mu_{C_{t_{0}}}\left(F_{t_{r / 0}}^{N e}\right)-\mu_{C_{t_{0}}}\left(F_{t_{1 / 0}}^{N e}\right)\right)
$$

where $N_{r}$ is the number of time measurements between $t_{0}$ and $t_{k} . \quad \mu_{C_{t_{0}}}\left(F_{t_{r / 0}}^{N e}\right)$ (respectively $\mu_{C_{t_{0}}}\left(F_{t_{1 / 0}}^{N e}\right)$ ) denotes the average pixel intensity in the interest area of $F_{t_{r} / 0}^{N e}$ (respectively $F_{t_{1 / 0}}^{N e}$ ) delimited by $C_{t_{0}}$. If the normalised data with Eq. (10) does not respect the rare event hypothesis, the calculated statistics by Eq. (9) will then be biased. Nevertheless, we do not use the absolute values of these statistics but their spatial repartition.

\section{CHANGE CRITERION}

The homogeneity map $S M_{t_{k}}$ can be integrated into a scalar criterion in order to get a treatment efficacy quantification. To do so, we firt define a function $f_{s}(p)$ that represents the spatial repartition of the changes by:

$$
f_{s}(p)=\frac{\operatorname{card}\left\{x: S M_{t_{k}}(x) \leq p\right\}}{\operatorname{card}\left\{S M_{t_{k}}\right\}} .
$$

$f_{s}(p)$ is an increasing function from $[0,1]$ to $[0,1]$. As the interpretation of $f_{s}(p)$ is not straightforward, we prefer to work with $f_{t_{k}}^{p}(s)$ that is the inverse function of $f_{s}(p)$. A linear interpolation is used to calculate $f_{t_{k}}^{p}(s)$ with a regular sampling along $s . f_{t_{k}}^{p}(s)$ is also an increasing function from $[0,1]$ to $[0,1]$. Then, an integration of $f_{t_{k}}^{p}(s)$ to a scalar $H_{t_{k}}$ is computed by:

$$
H_{t_{k}}=2 \int_{0}^{1}\left|f_{t_{k}}^{p}(s)-s\right| d s .
$$

$H_{t_{k}} \in[0,1]$ represents the area between the function $f_{t_{k}}^{p}(s)$ and the identity function $\operatorname{Id}(s)=s$. $I d$ corresponds to the most heterogeneous repartition that can be expected. Then, the higher $H_{t_{k}}$ is, the more homogeneous changes are. We use the Riemann integral to calculate $H_{t_{k}}$ with discrete data. The homogeneity measure of Eq. (12) makes sense only when some changes appear in the image between $t_{0}$ and $t_{k}$. We estimated that, if less that $10 \%$ of the ROI change between $I_{0}$ and $I_{k}$, the criterion $H_{t_{k}}$ cannot be interpreted.

\section{EXPERIMENTAL RESULTS}

This section shows results obtained by the proposed method on patients taken from a clinical study involving melasma. We used the method proposed in [3] to obtain the spectral signature that gives $I_{k}$, and used a SVM classification to calculate $M_{p_{0}}$. Then, the images series $I_{0}, \ldots I_{n}$ were co-registered with the algorithm proposed in [7]. To smooth $C_{k}$ we use a 13 pixel diameter Gaussian kernel. The chosen family of thresholds is $U=[1,1.5,2,2.5,3]$ for all studied images. This

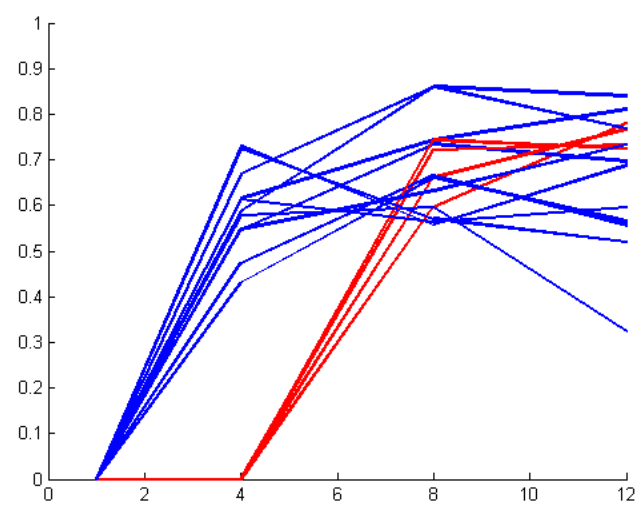

Fig. 1. Time sequences of $H$ for patients whose pathology spatially decrease by more than $10 \%$. $\mathbf{X}$-axis: time in weeks $\left(t_{0}=1, t_{1}=4, t_{2}=8, t_{3}=12\right)$, Y-axis: $H$ value.

thresholds family has been manually selected on a subset of images. Figs. 2 and 3 show two examples of obtained maps for two patients' time series. Fig. 2 corresponds to a patient where dermatologist diagnoses a significant change whereas Fig. 3 corresponds to a patient whose diagnosis reveals few changes. As one can see, the proposed method allows us to detect precisely the areas where a change appears. Moreover, we can distinguish at an earlier stage non significant changes (in red) that can become significant later (in blue). We ran the computation of $H$ on a series of 22 patients under a treatment. The clinical analysis of this population concludes that the treatment had globally an effect. In the population of 22 patients, we observed that for 7 patients the criterion $H$ is not interpretable. Indeed, for five patients less than $10 \%$ of the ROI changes at time $t_{2}=8$ or $t_{3}=12$ and for two patients there were less than $10 \%$ of the ROI that change in all the time sequence. For the others fifteen patients (see Fig. 1) we observe an global growth of $H$ with time. For most patients (in blue) main changes occurred in the second measurement time $t_{1}=4$, and for 4 patients (in red), main changes occurred in time $t_{2}=8$. These results show that the homogeneity criterion gives relevant interpretation of disease progression during treatment.

\section{CONCLUSION}

In this paper, we adapt the SPM methodology to skin lesion analysis. Associated with a classification and a spectral signature, it gives a precise map of the changes appearing during the treatment phase. For an individual patient, the proposed method gives a spatial analysis of the changes. As the proposed method is automatic, the severity estimation is more robust in a time sequence than a human inspection. For the change detection to succeed, both co-registration between time measurements and classification of the ROI should be accurate. 


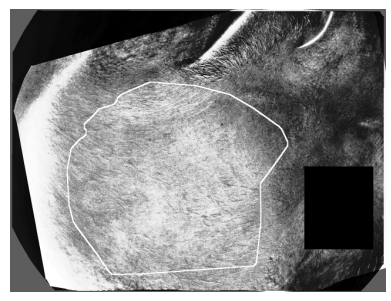

(a)

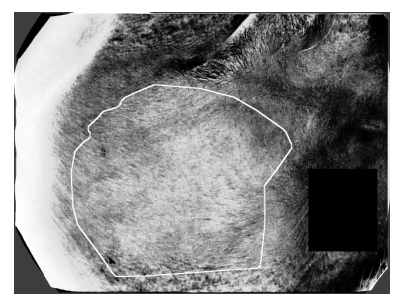

(c)

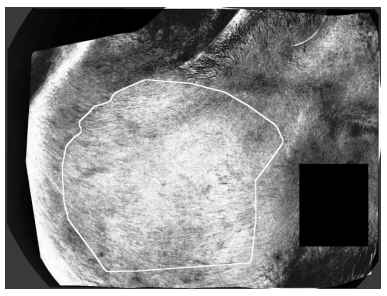

(e)

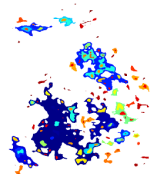

(b)

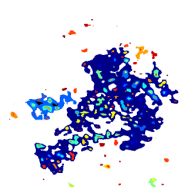

(d)

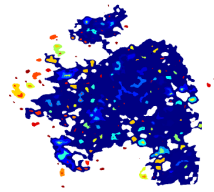

(f)

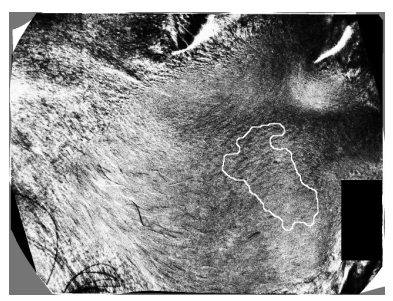

(a)

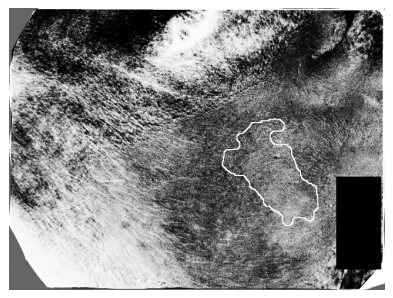

(c)

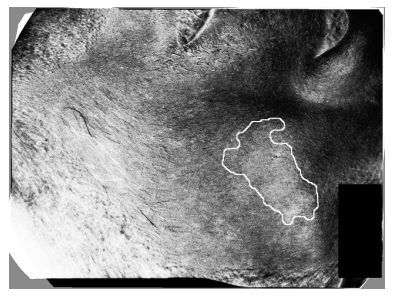

(e)

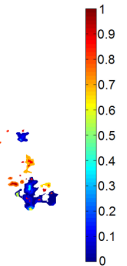

(b)

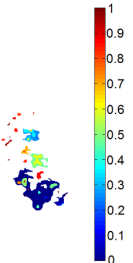

(d)

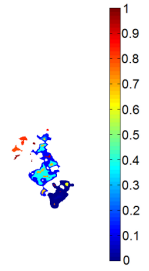

(f)

Fig. 2. Change maps obtained for a patient whose pathology decreased during the treatment period. a,c,e) equalised difference images on the spectral feature maps between each time $t_{1}, t_{2}$ et $t_{3}$ and $t_{0}$. b,d,f) homogeneity maps $S M_{t_{k}}$ for the three time $t_{1}, t_{2}$ et $t_{3}$ using the normalisation of Eq. (10).

\section{ACKNOWLEDGEMENT}

The authors would like to thank Dr. Philippe Martel and Ms. Anne-Sophie Dugaret for interesting discussions, and Galderma $R \& D$ for partial funding and for providing the data.

\section{REFERENCES}

[1] G. N. Stamatas, B. Z. Zmudzka, N. Kollias, and J. Z. Beer, "In vivo measurement of skin erythema and pigmentation: new means of implementation of diffuse reflectance spectroscopy with a commercial instrument," British Journal of Dermatology, vol. 159, pp. 683-690, 2008.

[2] S. Prigent, X. Descombes, D. Zugaj, P. Martel, and J. Zerubia, "Multi-spectral image analysis for skin pigmentation classification," in Proc. IEEE International Conference on Image Processing (ICIP), Hong-Kong, China, September 2010.

[3] S. Prigent, D. Zugaj, X. Descombes, P. Martel, and

Fig. 3. Change maps obtained for a patient whose pathology decreased during the treatment period. a,c,e) equalised difference image on the spectral feature maps between each time $t_{1}, t_{2}$ et $t_{3}$ and $t_{0}$. b,d,f) homogeneity maps $S M_{t_{k}}$ for the three time $t_{1}, t_{2}$ et $t_{3}$ using the normalisation of Eq. (10).

J. Zerubia, "Estimation of an optimal spectral band combination to evaluate skin disease treatment efficacy using multi-spectral images," in Proc. IEEE International Conference on Image Processing (ICIP), Brussels, Belgium, September 2011.

[4] K.J. Friston, J. Ashburner, S.J. Kiebel, T.E. Nichols, and W.D. Penny, Eds., Statistical Parametric Mapping: The Analysis of Functional Brain Images, Academic Press, 2007.

[5] R.J. Alder, The Geometry of Random Fields, Wiley, 1981.

[6] J.B. Poline, K.J. Worsley, A.C. Evans, and K.J. Friston, "Combining spatial extent and peak intensity to test for activations in functional imaging," NeuroImage, vol. 5, no. 2, pp. 83-96, 1997.

[7] A. Myronenko and X. Song, "Intensity-based image registration by minimizing residual complexity," IEEE Trans. on Medical Imaging, vol. 29, no. 11, pp. 18821891, 2010. 